\title{
Dark Matter from Symmetry
}

\author{
W. Chagas-Filho \\ Physics Department, Federal University of Sergipe, SE, Brazil \\ Email: wfilho@ufs.br
}

\begin{abstract}
We present the idea that a reasonable explanation for dark matter and dark energy can be obtained from the reparametrization symmetry of the action integral that describes a scalar relativistic particle.
\end{abstract}

Keywords: Dark matter; dark energy; reparameterization invariance; relativistic particles.

\section{Introduction}

Today there are astronomical observations that support the idea that our Universe also contains unknown forms of matter and energy which are now called dark matter and dark energy. In particular, dark matter should repel the usual matter, and this would explain the observed rate of expansion of the Universe. Several models have been proposed to describe these dark versions of matter and energy. However their physical origin remains a mystery.

In this letter we present a simple perspective that may be used to explain the possible existence of dark matter and dark energy. According to our perspective, dark matter would simply be negative matter and dark energy would simply be negative energy.

To start explaining our point of view, let us recall that there is nothing wrong with the concept of a negative energy. For instance, a planet in an elliptic orbit in Newtonian gravitation has a negative total energy [1]. A relativistic electron in the Hydrogen atom, described by Quantum Mechanics, has a negative total energy [2]. Negative energies have been present in Physics for a long time. They are usually associated with the physical notion of bounded motion. On the contrary, negative masses have never been found.

In this letter we show that a negative mass must emerge if we want to extend the reparameterization symmetry of the action for a relativistic particle to include also discontinuous reparameterizations According to this result, a relativistic particle with a negative mass propagates backwards in proper time, but the classical action describing the particle can not distinguish between backward and forward propagation.

\section{Relativistic Particles}

The action for a scalar relativistic particle is the simplest example of the action for a general covariant system. It is a prototype for String Theory and contains the basic conceptual features of General Relativity. The action is proportional to the length of the world-line traveled by the particle, with the particle's mass $m$ as the constant of proportionality

$$
S=m \int \mathrm{d} s
$$

where $d s$ is the interval between two infinitesimally close space-time events, given by

$$
\begin{aligned}
d s^{2} & =c^{2} \mathrm{~d} t^{2}-\mathrm{d} x^{2}-\mathrm{d} y^{2}-\mathrm{d} z^{2} \\
& =\eta_{\mu \nu} \mathrm{d} x^{\mu} \mathrm{d} x^{\nu}=\mathrm{d} x^{\mu} \mathrm{d} x_{\mu}
\end{aligned}
$$

where $x^{\mu}=\left(x^{0}, x^{1}, x^{2}, x^{3}\right)=(c t, x, y, z)$ are the coordinates of space-time and $\eta_{\mu \nu}=\operatorname{diag}(+1,-1,-1,-1)$ is the flat space-time metric. 
Using (2.2) the action (2.1) becomes

$$
S=m \int \sqrt{\mathrm{d} x^{\mu} \mathrm{d} x_{\mu}}
$$

Introducing the particle's proper time $d \tau=\frac{d s}{c}$ as a parameter along the particle's world-line, we can rewrite action (2.3) as

$$
S=m \int \mathrm{d} \tau \sqrt{\frac{\mathrm{d} x^{\mu}}{\mathrm{d} \tau} \frac{\mathrm{d} x_{\mu}}{\mathrm{d} \tau}}
$$

\section{Reparameterization Symmetry}

Written in the form (2.4), the action for the relativistic particle is invariant under the transformation

$$
\tau \rightarrow \bar{\tau}=f(\tau)
$$

where $f(\tau)$ is an arbitrary continuous function with an inverse $f^{-1}(\tau)$. This is because in this case

$$
\begin{gathered}
\mathrm{d} \bar{\tau}=\frac{\mathrm{d} f}{\mathrm{~d} \tau} \mathrm{d} \tau \\
\frac{\mathrm{d} x^{\mu}}{\mathrm{d} \bar{\tau}}=\left(\frac{\mathrm{d} f}{\mathrm{~d} \tau}\right)^{-1} \frac{\mathrm{d} x^{\mu}}{\mathrm{d} \tau}
\end{gathered}
$$

and action (2.4) transforms as

$$
\begin{aligned}
S \rightarrow \bar{S} & =m \int \frac{\mathrm{d} f}{\mathrm{~d} \tau} \mathrm{d} \tau \sqrt{\left(\frac{\mathrm{d} f}{\mathrm{~d} \tau}\right)^{-1} \frac{\mathrm{d} x^{\mu}}{\mathrm{d} \tau}\left(\frac{\mathrm{d} f}{\mathrm{~d} \tau}\right)^{-1} \frac{\mathrm{d} x_{\mu}}{\mathrm{d} \tau}} \\
= & m \int \frac{\mathrm{d} f}{\mathrm{~d} \tau} \mathrm{d} \tau \sqrt{\left(\frac{\mathrm{d} f}{\mathrm{~d} \tau}\right)^{-2} \frac{\mathrm{d} x^{\mu}}{\mathrm{d} \tau} \frac{\mathrm{d} x_{\mu}}{\mathrm{d} \tau}} \\
= & m \int \frac{\mathrm{d} f}{\mathrm{~d} \tau} \mathrm{d} \tau\left(\frac{\mathrm{d} f}{\mathrm{~d} \tau}\right)^{-1} \sqrt{\frac{\mathrm{d} x^{\mu}}{\mathrm{d} \tau} \frac{\mathrm{d} x_{\mu}}{\mathrm{d} \tau}} \\
& m \int \mathrm{d} \tau \sqrt{\frac{\mathrm{d} x^{\mu}}{\mathrm{d} \tau} \frac{\mathrm{d} x_{\mu}}{\mathrm{d} \tau}}
\end{aligned}
$$

being therefore invariant under the transformation (2.5).

However, the symmetry (2.5) breaks down if we consider the discontinuous transformation

$$
\tau \rightarrow \bar{\tau}=-\tau
$$

In this case action (2.4) transforms as

$$
\begin{gathered}
S \rightarrow \bar{S}=m \int(-\mathrm{d} \tau) \sqrt{\frac{\mathrm{d} x^{\mu}}{\mathrm{d} \tau} \frac{\mathrm{d} x_{\mu}}{\mathrm{d} \tau}} \\
=-m \int \mathrm{d} \tau \sqrt{\frac{\mathrm{d} x^{\mu}}{\mathrm{d} \tau} \frac{\mathrm{d} x_{\mu}}{\mathrm{d} \tau}}
\end{gathered}
$$

that is, the action changes sign.

This same sign change occurs in the tetrad formulation of General Relativity, with action

$$
S=\frac{1}{2} \int e^{I} \wedge e^{J} \wedge F^{K L} \epsilon_{I J K L}
$$

where

$$
F^{I J}=e_{\mu}^{I} e_{\nu}^{J} R_{\rho \sigma}^{\mu \nu} \mathrm{d} x^{\rho} \wedge \mathrm{d} x^{\sigma}
$$


under the internal time reversal operation

$$
T e^{0}=-e^{0} \quad T e^{i}=e^{i} \quad i=1,2,3
$$

See reference [3] for details.

However, contrary to the case in General Relativity (which can not explain the existence of dark matter) here we can restore the invariance of the action (2.4) under the discontinuous transformation (2.8) by postulating that the correct transformation is

$$
\tau \rightarrow \bar{\tau}=-\tau \quad m \rightarrow-m
$$

Therefore we have obtained a theoretical evidence for negative masses by simply requiring that the relativistic particle action (2.4) be invariant under discontinuous reparameterizations.

If we now consider Newton's equation for the attractive gravitational force between two masses $m$ and $M$ separated by a distance $r$

$$
\boldsymbol{F}=-G \frac{m M}{r^{2}} \boldsymbol{u}_{r}
$$

and substitute $m$ by $-m$, the equation becomes

$$
\boldsymbol{F}=+G \frac{m M}{r^{2}} \boldsymbol{u}_{r}
$$

That is, the gravitational interaction between a positive and a negative mass is repulsive, exactly as the astronomical observations suggest in the case of dark matter. Furthermore, if we consider Einstein's equation $E=m c^{2}$ and substitute $m$ by $-m$ we get $E=-m c^{2}$. From this perspective the existence of dark energy is a consequence of the existence of dark (negative) masses.

\section{Concluding Remarks}

It may be interesting to try to construct a picture of the universe using only the ideas introduced in this paper. The first point is that if we take a negative mass $-m$ and another negative mass $-M$ and compute the force due to their gravitational interaction using Newton's equation (2.14) we find

$$
\begin{aligned}
\boldsymbol{F}= & -G \frac{(-m)(-M)}{r^{2}} \boldsymbol{u}_{r} \\
& =-G \frac{m M}{r^{2}} \boldsymbol{u}_{r}
\end{aligned}
$$

which is identical to (2.14). Therefore the gravitational force between two negative masses is an attractive force. As is the gravitational force between two positive masses. Based on this fact we may expect that all the structures that are formed by the attractive gravitational force between positive masses can also be formed by the attractive gravitational force between negative masses.

The second point is that the structures formed by negative masses will repel the structures formed by positive masses and vice-versa. This provides the extra gravitational force to allow structures to form more quickly.

The third point is that, if we assume that the Big Bang hypothesis is correct, then there must exist equal amounts of positive and negative matter in the universe. The present-day observed predominance of negative matter over positive matter may then be just a temporary non-homogeneity of the part of the universe in which we live. According to this point of view it is possible that, in the future, the attractive gravitational forces between masses with the same sign and the repulsive gravitational forces between masses with opposite signs will bring the universe to a stable homogeneous configuration.

\section{References}

1. M. Alonso and E. J. Finn, Fundamental University Physics, Addison Wesley Publishing Co. 1967

2. R. Eisberg and R. Resnick, Quantum Physics, John Wiley and Sons, Inc. 1974

3. C. Rovelli and F. Vidotto, Covariant Loop Quantum Gravity, Cambridge University Press 2015 